\title{
On the Geometry of Random Cantor Sets and Fractal Percolation ${ }^{1}$
}

\section{K. J. Falconer ${ }^{2}$ and G. R. Grimmett ${ }^{3}$}

Received June I, 1993

Part a of Theorem 1 is incorrect as stated, and its hypothesis must be strengthened in the following way. For $\mathbf{k} \in J^{e}=\{0,1, \ldots, M-1\}^{e}$, let

$$
N(\mathbf{k})=\left|\left\{\mathbf{i}=\left(i_{1}, \ldots, i_{d}\right) \in X:\left(i_{1}, \ldots, i_{e}\right)=\mathbf{k}\right\}\right|
$$

the number of cubes contributing to $C_{1}$ which project onto the subcube of $[0,1]^{e}$ corresponding to the vector $\mathbf{k}$. We set $v=\min \left\{N(\mathbf{k}): \mathbf{k} \in J^{e}\right\}$, and note that $m(\mathbf{k})=E(N(\mathbf{k}))$.

Theorem 1, part a, should be replaced by the following; the other parts of the theorem are correct as stated.

Part a of Theorem 1. Suppose $m>1$. Then

$$
P\left(\pi_{e} C \text { contains a ball } \mid C \neq \phi\right)=1
$$

and

$$
P\left(\pi_{e} C=[0,1]^{e}\right)>0
$$

if and only if either $P(v \geqslant 1)=1$ or $P(v \geqslant 2)>0$.

In the case when the generating measure $\mu$ is product measure (see lines 6-12 on page 468), we have that $P(v \geqslant 2)>0$, whence the conclusion of part a applies if $m>1$.

There is exactly one (minor) consequence of this correction to the further contents of the paper. The final sentence of p. 467 should now read as follows:

\footnotetext{
${ }^{1}$ Journal of Theoretical Probability 5, 465-485 (1992).

${ }^{2}$ School of Mathematics, University Walk, Bristol BS8 1TW, United Kingdom.

${ }^{3}$ Statistical Laboratory, 16 Mill Lane, Cambridge CB2 1SB, United Kingdom.
} 
"In the case $m=1$, conclusions (1.3) and (1.4) hold if and only if (i) $\operatorname{var}(N(\mathbf{k}))=0$ for all $\mathbf{k}\left(\in J^{e}\right)$ such that $m(\mathbf{k})=1$, and (ii) either $P(v \geqslant 1)=1$ or $P(v \geqslant 2)>0$."

In the light of the amended version of Theorem 1, the proof of this remark holds as before.

Proof of Part a. The proof given is incorrect at line 19 of p. 470, where it is assumed that $P\left(A_{r}\right)>0$ for all $r$. Certainly this is valid if $1<\eta \leqslant 2$ and $P(v \geqslant 2)>0$, since $P\left(A_{1}\right) \geqslant P(v \geqslant 2)$ and

$$
P\left(A_{r} \mid A_{r-1}\right) \geqslant P(\nu \geqslant 2)^{(2 M)^{r-1}} \quad \text { for } r \geqslant 2
$$

Secondly, if $P(v \geqslant 1)=1$ then it is immediate that $P\left(\pi_{1} C=[0,1]\right)=1$. Finally suppose the converse, that

$$
0<P(v=0)=1-P(v=1)
$$

Now $\mu_{n} \geqslant 1$ if and only if $N(\mathbf{i}) \geqslant 1$ for all $\mathbf{i} \in J^{m}$ and all $1 \leqslant m<n$. We have that $P(v \leqslant 1)=1$, and it follows by induction on $n$ that

$$
P\left(\mu_{n}>0\right) \leqslant P(v \geqslant 1)^{n}
$$

which tends to 0 as $n \rightarrow \infty$, by (*). Hence Eq. (1.4) is false. By an argument similar to the proof of part b, one finds that Eq. (1.5) holds under (*).

\section{ACKNOWLEDGMENT}

We are indebted to Michel Dekking for his having read our paper, for finding the error, and for proposing this correction. 